\title{
Precision timing options for the Apple Macintosh family of computers
}

\author{
JAMES M. KIELEY \\ Claremont Graduate School, Claremont, California \\ and \\ TIMOTHY S. HIGGINS \\ Pomona College, Claremont, California
}

\begin{abstract}
Several software development systems for the Macintosh are examined for their suitability for real-time control of experiments. New methods of millisecond resolution timing with Pascal and $\mathrm{C}$ languages are provided, along with routines for timing within BASIC with tick-level accuracy. Each of the routines presented here should work equally well with the Macintosh Plus, SE, and II, and with future models that Apple may introduce.
\end{abstract}

A number of papers appearing in the last few years have focused on the expanding role of the Apple Macintosh microcomputer in the laboratory setting. It has been pointed out that the Macintosh offers advantages over many competing microcomputers because of its powerful built-in graphics routines and an easy user interface. A shortcoming of the Macintosh for use in research applications has been its lack of hardware expandability. Apple has provided a solution to this problem with the introduction of two new models in 1987, the Macintosh SE and the Macintosh II, each of which offers one or more internal slots for add-on hardware devices similar to those seen in other popular laboratory computers, such as the IBM PC and the Apple II.

A second obstacle faced by researchers using the Macintosh has been the problem of accurately timing very brief events. Westall, Perkey, and Chute (1986) presented the Drexel MilliTimer, which was designed to allow millisecond accuracy timing of events within a variety of programming environments. Lane and Ashby (1987) provided an entire library of machine language routines intended for use with Microsoft BASIC. Included in this collection is a routine for presenting graphical images and/or sounds and recording keystroke response times with reported millisecond accuracy. A problem common to these timing routines is that they only work with some Macintosh models. Each timer is address-dependent and may cause a system crash when run on the Macintosh SE or II.

Since its introduction in 1984, the Macintosh has gone through a rapid evolution, resulting in at least four distinct versions of the ROM chips that contain much of the code that gives the computer its distinctive features. The first Macintosh came with only 64K ROM and was relatively limited in the number of special ROM Toolbox rou-

Correspondence may be sent to James M. Kieley, Benezet Psychology Building, Claremont Graduate School, Claremont, CA 91711. tines it contained. (Apple uses the term Toolbox to refer to the library of internal procedures and functions that can be accessed from within programs.) Two later versions of the Macintosh, including the Macintosh Plus, came with 128K ROM, within which additional system information and Toolbox routines were added as other code was relocated. The newer Macintosh II and SE models come with 256K ROM, although there are significant ROM additions unique to the Macintosh II that allow it to support color displays, multiple add-on devices, and enhanced sound capabilities.

As was suggested before, the only way to do millisecond timing with the early models of the Macintosh was to write an address-specific routine in assembly or machine language that could access one of the timers on the Versatile Interface Adapter (VIA) chip, which controls the clock, keyboard, mouse, sound driver, and other internal signals (see Westall et al., 1986, for details). The desirability of this approach is limited by (1) the fact that different address-specific routines need to be constructed for the various ROM versions in machines already in existence and for those that will be introduced in the future and (2) the prerequisite ability to program proficiently in Motorola 68000 source code. Westall et al. (1986) have discussed the fact that the early versions of software development tools for the Macintosh (with the exception of Rascal, discussed later) provided no simple and direct way to carry out millisecond accuracy timing. More striking is the fact that certain programming languages, notably Microsoft BASIC 3.0, provide no documented support for timing with even a tick (approximately $16.625 \mathrm{msec}$ ) level of resolution.

The primary purpose of this paper is to critically review the timing options possible with the software development systems currently available for the Macintosh and to offer new general-purpose solutions for accurate timing on these machines. The accompanying appendices list routines that are similar in function, written in the popular languages 
BASIC, Pascal, and C. Each of these timers can be set up as an interval timer (ITIMER), which is appropriate for invoking a delay in program execution that is more accurate than cycle-counting timing loops (see Dorfman, 1987 , for a discussion of various approaches that can be taken to conduct software-based timing). Alternatively, each of the timers can be modified to record reaction time to make a keypress response after a stimuius presentation. Each reaction-time timer (RTimer) contains code that initially flushes out the computer's event queue in order to prevent prestimulus keypresses still in memory from falsely triggering the timer. Each RTimer listing also contains code that synchronizes the presentation of a stimulus with the beginning cycle in the screen vertical retrace sequence. This technique ensures that each stimulus presentation begins at the same relative point in time, immediately after the tick counter has been incremented (see Westall et al., 1986). The $\mathrm{C}$ and Pascal routines shown in the appendices are machine-independent in that they operate off labeled ROM calls that are included in the Macintosh Toolbox. Since these routines rely on labeled internal procedures rather than addresses, and it has been Apple's policy to continue to support old ROM calls with new versions of the operating system, it is relatively safe to assume the timers will continue to work with future versions of the Macintosh. For BASIC programmers, we first briefly discuss BASIC language products and then describe a simple means of interval and response timing that will, on average, be accurate $( \pm 8 \mathrm{msec})$.

\section{Timing in BASIC}

Several versions of BASIC for the Macintosh are currently available. Among the most popular are Microsoft Basic, ZBASIC, and True Basic. It has been suggested that BASIC is the language of choice among psychologists who have limited time to master the intricacies of more advanced languages (Lehman, 1988). BASIC's reduced instruction set is often adequate for generalpurpose laboratory software, and program debugging is relatively simple and straightforward. The BASICs available for the Macintosh generally fall into the category of what has been termed structured BASIC. Each of the three previously mentioned products has advanced features that allow for Pascal-like repeat loops, and each permits partial access to the Macintosh Toolbox. ZBASIC provides documented supported for the TickCount ROM function, which can be called to return the number of ticks that have elapsed since system start-up. (ZBASIC also has a built-in delay function that is inaccurately documented in its user's manual as being able to suspend program execution with millisecond precision. In actuality, this built-in function provides no better than tick-level [ $\pm 16 \mathrm{msec}$ ] delay accuracy.) Support for the Toolbox TickCount function is undocumented in the Macintosh's Microsoft Basic and True Basic manuals. However, tick timing is possible by reading two addresses using a BASIC PEEK command. These addresses are incremented at different intervals dur- ing the vertical retrace interrupts that occur every $16.6 \mathrm{sec}$ the machine is running. PEEK(365) provides an 8-bit counter that is incremented during each interrupt and that will keep track of the number of 16.6-msec ticks that have elapsed up until the counter turns over. PEEK(364) provides an 8-bit counter that is incremented each time the location represented by PEEK(365) turns over. Since each tick actually lasts $16.625822 \mathrm{msec}$, it takes PEEK(365) about $4.2562 \mathrm{sec}$ to roll over, starting from zero. Starting from zero, PEEK(364) rolls over every $1089.589 \mathrm{sec}$, which means that after being set to zero these two addresses can provide a fairly accurate timer for durations of up to $18.15983 \mathrm{~min}$. This method of timing is independent of CPU clock speed and works functionally the same on the 16-MHz Motorola-68020-based Macintosh II and the 8-MHz Motorola-68000-based Macintosh Plus and SE.

The first section in Appendix A provides a listing for a BASIC subroutine that can be used for interval timing accurate to the near-tick level. The second listing in Appendix A shows how the routine can be modified to record reaction time to make a keypress after a stimulus has been presented. As discussed in Inside Macintosh (Apple Computer, 1985), the definitive technical reference for the Macintosh, some degree of caution must be used when relying on tick counts for accurate timing since certain types of event sequences may prevent normal vertical retrace interrupts. Generally speaking it can be assumed that the tick counter will be reliably incremented only during circumstances in which no disk-drive access is taking place and the sound driver is not being accessed. Under these conditions, the tick count will change during the 1.2-msec vertical blanking interval that occurs between video scans. A related matter, which we have previously mentioned, concerns the error (average of 8.33 msec) that can be introduced into reaction-time studies if stimulus presentations are not synchronized with the screen vertical retrace. The RTimer routine shown in Appendix A waits until the tick counter, which has been previously set to zero, is incremented by 1 before presenting a stimulus. This procedure synchronizes the beginning of the stimulus event presentation with the beginning of the vertical retrace cycle.

The BASIC routines listed here have been tested on a Macintosh Plus, SE, and II, and have been found to work reliably on all three machines. They are independent of CPU clock speed. Each of the BASIC language products mentioned above provides the user with the ability to produce compiled code (in some cases, at an additional expense) that reduces the speed of execution constraints normally associated with BASIC interpreters. However, none of these products provides the full Toolbox support necessary to produce timers with millisecond precision that will work with the various Macintosh models.

\section{Millisecond Timing in Pascal and $\mathbf{C}$}

Pascal and $\mathrm{C}$ are the languages of choice for the production of professional-grade application software for the 
Macintosh. Most prominent among the currently available development products is the Macintosh Programmer's Workshop (MPW), which was produced by Apple. MPW is a high-level development system that comes standard with an assembler. Shells for programming in Pas$\mathrm{cal}$ and $\mathrm{C}$ are available at an additional price. Two other popular choices among professionals who program on the Macintosh are Lightspeed C and Lightspeed Pascal, both marketed by Symantek. Other full-development systems for the Macintosh include TML Pascal and Aztec C. Each of these products claims to provide full support for over 700 ROM routines, which can be called within a program from the Macintosh Toolbox. Included among these routines are those in the Time Manager. The Time Manager procedures first appeared, with the introduction of the Macintosh Plus, in Inside Macintosh (Vol. IV, 1986). Unfortunately, the information in Inside Macintosh is not always easy to decipher under the best of conditions, and the section on the Time Manager is made more difficult because of an error: The tmCount field in the TMTask record is listed in the manual as a variable of type integer, when in fact it is a variable of type long integer. This difference is important because it allows millisecond timing to be extended for periods much longer than what would be possible if the information in Inside Macintosh were correct (approximately 24 days as opposed to $30 \mathrm{sec})$. In any case, the routines in the Time Manager provide the support for millisecond-level event timing that was very difficult with earlier versions of the operating system and ROM. We previously discussed the advantages of having timing routines accessible as labeled procedures rather than as low-level address-specific locations only reachable with assembly language. The Time Manager provides this level of support, which reduces the need to rely on model-specific assembly language routines that may crash the first time they find something in an unexpected location in memory.

The listings in Appendices B and C show routines written in MPW Pascal and MPW $C$ that use the Time Manager and that can operate in a manner roughly analogous to the ITimer and RTimer shown in Appendix A. The margin comments attached to these two listings suggest how the code can be modified to work as either an ITimer or an RTimer. Although these routines are functionally similar to the BASIC routines, there are important differences. Rather than counting up the number of ticks that occur after timer initialization, the Time Manager-based routines count down a millisecond at a time from a previously specified value until they either detect a keypress or reach zero. Similar to the BASIC example, the Pascal and $\mathrm{C}$ timers use a tick counter to synchronize stimulus presentation and timer initialization with the beginning of the screen vertical refresh cycle. However, in Pascal or $\mathrm{C}$, the tasks of first clearing out the keyboard buffer and then determining when the tick counter has been incremented can be conveniently handled by ROM calls to FLUSHEVENTS and TICKCOUNT.
The routines in Appendices B and C are, in theory, able to provide timing resolution that is 16 times greater than that provided by a tick-based timer. We have tested this theory and found it to be correct when the routines are used to initiate delays in program execution. However, use of the Time Manager may not be a suitable method to conduct timing in every reaction-time gathering situation due to variable delays that occur between the time an interrupt is generated from input devices (e.g., keyboards and mouse buttons) and when an interrupt is passed on to the operating-system event manager. In an ideal situation, the Macintosh operating system's event manager checks to see if there has been a keypress at the end of each vertical retrace cycle, which occurs every $16.6 \mathrm{msec}$. In practice, if either the sound driver, a disk drive, the memory manager, or a number of other things are being accessed, a normal vertical blanking interrupt might be skipped altogether, thus magnifying what might be termed interrupt error. Even if a programmer is careful to avoid this kind of pitfall, he can assume that the time for an interrupt to be detected will vary about $8 \mathrm{msec}$ across trials if the normal method of posting events is being used. The only way to circumvent this built-in limitation is to poll the VIA chip directly (see Gillespie, 1985; Westall et al., 1986) using assembly- or machine-language routines. Keep in mind that VIA-based timers must be modified to work properly on the newer Macintosh models. One way to go about this, and still maintain compatibility with all Macintosh models, is to build a single timing library with submodules that set up the timer for a specific model's ROM version and CPU clock speed. Apple has made the programming of this type of timing library easier by including the ROM routine SysEnvirons, which can be used to identify model and CPU information from within an assembly-language program.

\section{Real-Time Programming in Rascal}

One other language product that deserves attention is Rascal (Reed College, 1986). Rascal, which is marketed by the MetaResearch Group, is a language system that has been developed with the needs of researchers clearly in mind. Rascal (a hybrid of Pascal and C) has numerous useful routines, including easy-to-use built-in functions that allow millisecond accuracy timing of events with screen synchronization. Version 2.0 of Rascal (reviewed here) has since been replaced by version 3.0. The newer version reportedly features expanded Toolbox support that includes access to the color-quickdraw routines that can be used on the Macintosh II. We used version 2.0 of Rascal to reproduce a millisecond timing demonstration shown in the Rascal manual. The program ran flawlessly on Macintosh Plus, SE, and II. The principal criticism that can be made of Rascal is that it is a complex nonstandard language that is time-consuming and difficult to learn. (The authors of the manual admit that "Rascal is somewhat more difficult to master than Pascal.") When it was first introduced, Rascal was the only real alterna- 
tive for those wishing to undertake real-time programming. However, with the addition of the Time Manager to the Macintosh Toolbox, limited millisecond timing is possible with such standard-language products as Pascal or $\mathrm{C}$.

\section{Deciding Which Language to Use}

This paper has attempted to point out that a variety of approaches can be taken when programming the Macintosh for experiments that depend on precise event timing. The first consideration to be addressed when deciding which route to take should be the degree of timing precision actually needed. What are the predicted effect sizes, and are they likely to be masked or distorted if there is an added 8- or 16-msec average fluctuation in timing accuracy across trials, subjects, and conditions?

A second consideration deals with the time and programming talents available to the researcher. Constructing assembly-language routines and programming proficiently in Pascal or $\mathrm{C}$ can be challenging on any computer and can be even more difficult on the Macintosh when first learning. A fairly intimate understanding of the special use of the concept of resources and knowledge of how to use the Macintosh Toolbox are necessary for all but the most trivial programs with these languages. By way of contrast, BASIC on the Macintosh can be approached in much the same way as it is on other machines. There are special features on the Macintosh available to BASIC programmers who want to use them, but, essentially, no special knowledge of the inner workings of the computer is necessary. As we have said, the limitation with BASIC is that fully implemented millisecond timing must rely on libraries written in assembly language or on poking in machine code.

A third factor to consider in choosing development software is the specific Macintosh model that will be used immediately and the likelihood that a newer, more powerful model will replace it in the future. Timing routines provided by Gillespie (1985), Westall et al. (1986), and Lane and Ashby (1987) may be effective solutions for experimenters programming in BASIC using the pre-1987 Macintosh models. Those interested in using the SE or Macintosh II may wish to adapt for their own uses some of our suggestions, including the routines in the appendices.

\section{REFERENCES}

Apple Computer. (1985). Inside Macintosh (Vol. 1-3). Menlo Park, CA: Addison Wesley.

APPLE CoMPUTER. (1986). Inside Macintosh (Vol. 4). Menlo Park, CA: Addison Wesley.

DoRFMAN, D. (1987). Software timing of events in cognitive psychology experiments. Behavior Research Methods, Instruments, \& Computers, 19, 185-190.

GILLESPIE, S. (1985). Millisecond timer mini-driver [Computer program]. Software and documentation available from DL-4 of MACPRO on CompuServe.

Lane, D. M., \& Ashby, B. (1987). PsychLib: A library of machine language routines for controlling psychology experiments on the Apple Macintosh computer. Behavior Research Methods, Instruments, \& Computers, 19, 246-248.

Lehman, R. S. (1988). The languages we use. Behavior Research Methods, Instruments, \& Computers, 20, 236-242.

REED COLLEGE. (1986). Rascal user manual: Macintosh language for real-time I/O oriented development. Portland, OR: Metaresearch.

Westall, R., Perkey, M. N., \& Chute, D. L. (1986). Accurate millisecond timing on Apple's Macintosh using Drexel MilliTimer. Behavior Research Methods, Instruments, \& Computers, 18, 307-311.

\section{APPENDIX A}

BASIC Timer

\section{ITimer}

REM The Basic interval timer can be called as a subroutine to instill fairly

REM precise $(+/-16.6 \mathrm{~ms})$ delays in program execution for up to 18.2 minutes.

REM S1 should be multiplied by 16.625 and S2 should be set to 0 (or omitted)

REM to calculate delays up to 4.239 seconds. To calculate longer delays

REM multiply S2 times 4256 and add that to S1 times 16.625 . This particular

REM version of the routine will produce a delay of 60 seconds.

ITimer:

$S 1=26: S 2=14$

POKE 364,0:POKE 365,0

$X 1=P E E K(365): X 2=P E E K(364)$ :WHILE $(X 1<S 1$ OR X2<S2) X1=PEEK(365):X2=PEEK(364):WEND

\section{RTimer}

REM This program clears out the keyboard buffer, waits for the vertical retrace REM interrupt before beginning to paint the screen with the stimulus, and then REM starts a timer. If no response occurs within 60 seconds the loop times out REM and repeats itself. If a space bar key press is detected time to respond REM to the nearest Tick is calculated and converted into milliseconds.

RTimer:

$S 1=26: S 2=14$ 
FLUSH:IF LEFT\$(INKEY\$,1) <> "' THEN FLUSH:REM Clear out keyboard buffer. POKE 364,0:POKE 365,0

WHILE PEEK(365)=0:WEND:REM Wait for vertical retrace interrupt 10 present stimulus. REM Present stimulus or do other processing here.

$\mathrm{X} 1=\operatorname{PEEK}(365)$ :X2=PEEK(364):WHILE (X1<S1 OR X2<S2) AND INKEY\$<>" "

$\mathrm{X} 1=\operatorname{PEEK}(365): \mathrm{X} 2=\operatorname{PEEK}(364):$ WEND

$\mathrm{RT}=(\mathrm{X} 2 * 256+\mathrm{X} 1-1) * 16.625: \mathrm{Rem} \mathrm{RT}=$ reaction time in converted to milliseconds

\section{APPENDIX B}

Pascal Timer

Type $\quad$ TMTaskPtr $=\wedge$ ${ }^{\wedge}$ TMTask;

Procedure MilliTimer;

Begin

End;

Procedure SetUpTimer(Timer: TMTaskPtr; Time: LongInt; Sync: Boolean);

Var LastTick: LongInt;

Begin

Timer^.tmAddr := @MilliTimer;

InsTime(Timer);

If $($ Sync $=$ TRUE) Then Begin

LastTick := TickCount;

Repeat Until (LastTick $<>$ TickCount);

End;

PrimeTime(Timer,Time);

End;

Procedure ShutDownTimer(Timer: TMTaskPtr);

Begin

RmvTime(Timer);

End;

Function DoTime(Length: LongInt; Sync: Boolean): LongInt;

Var OurTime: TMTask;

theEvent: EventRecord;

Done: Boolean;

Begin

Done := FALSE;

FlushEvents(everyEvent, 0 );

SetUpTimer(@OurTime,Length,Sync);

Repeat Begin

LastTime := OurTime.tmCount;

Done := GetOSEvent(keyDownMask, theEvent);

\{Delete this line if ITimer\}

End;

Until $($ Done $=$ True OR LastTime $=0) ;$ (Delete 'Done $=$ True OR' if ITimer $\}$

ShutDownTimer(@OurTime);

If (Done $=$ True) Then DoTime $:=$ LastTime;

Else DoTime $:=0$;

End; 


\section{APPENDIX C}

pascal void MilliTimer()

$\gamma^{*}$ This function is called by the Time Manager at the end of a Time Task */

1* Because there is no guarantee that the A5 world is correct when this */

/* routine is called, we cannot access any global variables from here. */

$1^{*}$ Therefore, it is up to the function that calls SetUpTimer to monitor */

$1 *$ the Time Manger Task to determine when the task is complete, and when */

$I^{*}$ to take the appropriate actions"/

\}

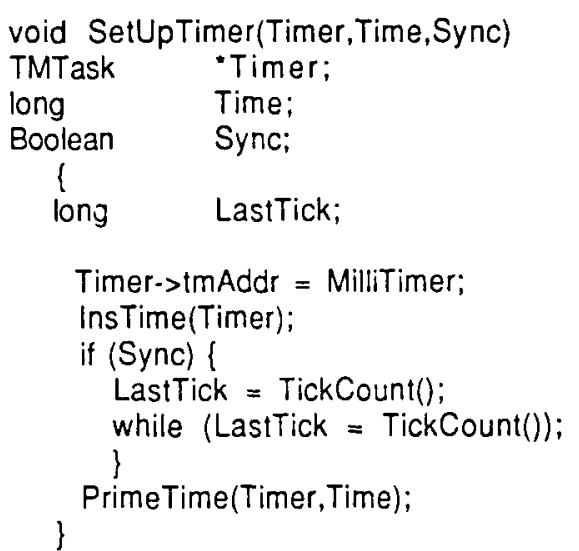

/* SetUpTimer accepts a pointer to a *

$l^{*}$ Time Manager Task, the duration in "/

$1 *$ Milliseconds of the task (a long), "/

${ }^{*}$ and a Boolean for requesting a *

$1 *$ Vertical Blank synchronization. */

1" The Task in initialized to point to "/

$l^{*}$ the MilliTimer Function (above), " $/$

$r^{*}$ and then is installed in the Time */

$1^{*}$ Manager Queue. If synchronization */

$l^{*}$ is requested, the routine reads the */

/* current TickCount, and delays until */

${ }^{*}$ TickCount has been changed by the */

1" Vertical Blank Interrupt */

$I^{*}$ Finally, the Task is started with a */

$I^{*}$ call to PrimeTime with the duration. */

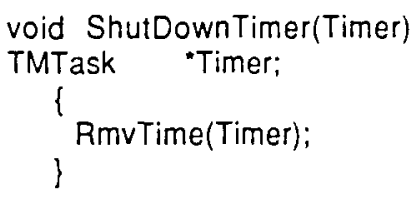

long DoTime(Length, Sync)

long Length;

Boolean Sync;

TMTask OurTime;

EventRecord the Event;

Boolean Done $=$ False

long LastTime;

FlushEvents(everyEvent,0);

SetUpTimer(\&OurTime,Length,Sync);

1* The User should add the actual object drawing at this point, so that it */

1 occurs just after the Time Manager Task has been set up. "/

while (IDone \&\&
LastTime $=$ OurTime.tmCount) \{

Done $=$ GetOSEvent $($ keyDownMask, \& the Event);

if (Done)

Last Time $=$ Length - LastTime; else LastTime $=0$;

ShutDownTimer(\&OurTime); return LastTime; \}
$1^{*}$ The while loop exits either when the */

1 * User presses a key, or when the Task' */

$1^{*}$ tmCount becomes zero (the Task has */

/" "Timed Out".) */

1* This Function accepts a pointer to */

$I^{*}$ a Time Manager Task that has been *

$l^{*}$ installed in the Time Manager Queue "

and removes it from the Queue. The */

$1^{*}$ This Function is an example of the *

1 * Use of the Time Manager. It takes */

1* a Millisecond duration (Length) and \%

I* a Request-Synchronization Boolean */

- OurTime is the Time Manger Task that

$1^{*}$ (tmCount is decremented every Milli-")

/ second by the Time Manager). */

1* Remove all Events from Event Queue */

$l^{*}$ Delete this and next line if interval */

$l^{*}$ timer (ITimer) function is desired *

$/^{*}$ If the User pressed a key the LastTime*/

$j^{*}$ is set the number of milliseconds it *

$1^{*}$ took for the key to be pressed, else */

$l^{*}$ Last time is set to zero. "

1 * The Time Task is removed from Queve */

$/ *$ and the value of LastTime is relurned */ 